\title{
Electrical Properties and Photo-response of FETs based on Few-layer $\mathrm{WS}_{2}$ Nanoflakes
}

\author{
Hang YANG ${ }^{1, a}$, Shi-Qiao QIN ${ }^{2, b}$, Fei WANG ${ }^{1, c}$, Jin-Yue FANG ${ }^{1, d}$ and Xue-Ao \\ ZHANG ${ }^{1, \mathrm{e}^{*}}$
}

${ }^{1}$ College of Science, National University of Defense Technology, Changsha 410073, China

${ }^{2}$ College of Optoelectronic Science and Engineering, National University of Defense Technology, Changsha 410073, China

ayanghangNUDT@163.com,, ${ }^{b}$ sqqin@nudt.edu.cn, ${ }^{c}$ wangfei_815@163.com, ${ }^{d}$ fjy_nudt@yahoo.com,

exazhang@nudt.edu.cn

*Corresponding author

Keywords: Few-layer $\mathrm{WS}_{2}$ nanoflakes, Field effect transistor (FET), Electrical properties, Photo-response

\begin{abstract}
The photo-electrical properties of few-layer $\mathrm{WS}_{2}$ nanoflakes, fabricated by mechanical exfoliation, were systematically studied in this paper. The few-layer $\mathrm{WS}_{2}$ FETs are n-type and possess a high gate modulation (On/Off ratio is larger than $10^{4}$ ) and a relatively high carrier mobility ( 7.110 $\left.0^{-3} \mathrm{~cm}^{2} \mathrm{~V}^{-1} \mathrm{~s}^{-1}\right)$. The photo-electrical properties of the device shows sensitive photo response, high photoresponsivity ( $\left.R_{\lambda}=3.5 \mathrm{~mA} / \mathrm{W}\right)$, quick response time $(t<20 \mathrm{~ms})$, high external quantum efficiency ( $\eta=0.68 \%$ ), and high detection rate $\left(D=1.7 \times 10^{9} W^{-1}\right)$ to red light $(638 \mathrm{~nm})$. These results showed that the extraordinary performance of the device based on few-layer $\mathrm{WS}_{2}$, which might open a new way to develop few-layer $\mathrm{WS}_{2}$-based material in the application of FETs and optoelectronics.
\end{abstract}

\section{Introduction}

Since graphene has been discovered by Geim and Novoselov in 2004[1], this two-dimensional (2D) material with typically extraordinary properties has attracted extensive research in the last few years[2-5]. Disappointingly, low On/Off ratio and low photoreponsivity has immensely restricted the application of this star material in logic transistor field and optoelectronics[6, 7].

Fortunately, atomically thin $\mathrm{WS}_{2}$, a new 2D material (transition-metal Dichalcogenide, TMD), has exhibited a remarkable On/Off ratio and a high mobility $[8,9]$. Compared to graphene, it may be more promising for the practical application in logical device. Besides the outstanding electrical properties, $\mathrm{WS}_{2}$ is a direct-bandgap semiconductor when exfoliated to monolayer state, which allow a high absorption coefficient under photoexcitation[10].

Although single-layer $\mathrm{WS}_{2}$ has been studied before[8, 11], to our knowledge, few has investigated the photo-electrical properties of few-layer $\mathrm{WS}_{2}(<5$ layers). Just as Geim said[12], even though monolayer graphene has plenty of excellent properties, bilayer and few-layer deserve carefully studying as well. Therefore, electrical properties and photo-response of FETs based on few-layer $\mathrm{WS}_{2}$ nanoflakes were systematically investigated in this paper.

Results indicate that few-layer has a relatively better photo-response than single-layer and multi-layer. More importantly, the complexity of the fabrication process of monolayer 2D material-based devices, such as thin-film transistors, limits their use[13]. Moreover, a further advantage of few-layer 2D material is its nearly degenerate direct and indirect bandgaps and high anti-photocorrosion stability[14, 15]. Accordingly, few-layer $\mathrm{WS}_{2}$ may possibly be more promising in the application of FETs and optoelectronics. 


\section{Experimental Details}

Device fabrication: The transistors based on few-layer $\mathrm{WS}_{2}$ were fabricated by standard micro-nano technology(EBL and EBE). Firstly, $\mathrm{WS}_{2}$ nanoflakes were exfoliated from the $\mathrm{WS}_{2}$ crystals(HQ graphene company) onto $300 \mathrm{~nm} \mathrm{SiO} / \mathrm{Si}$ substrates (doped $\mathrm{p}^{++}$, conductivity: $0.01-0.02 \Omega \cdot \mathrm{cm}$ ) using mechanical exfoliation technique[1]. Once $\mathrm{WS}_{2}$ nanoflakes were obtained, contacts were drawn using standard electron-beam lithography, and metallization was carried out with Ti/Au (20 $\mathrm{nm} / 70 \mathrm{~nm}$ ). Ti was chosen for the strong adhesion force with $\mathrm{SiO}_{2} / \mathrm{Si}$ substrate, however, may causing poor semi-metal contact. In devices, the layout of the electrical leads was designed to measure two-probe current-voltage characteristics.

Characterization and photo-electrical properties measurement: The $\mathrm{WS}_{2}$ nanoflakes were characterized by AFM to comprehend the thickness. The output and transfer electrical properties were measured by Keithley 4200 probe system (company: Cascade Microtech, model: Summit 11000B-M) in atmospheric environment and room temperature (300K). The photo-response of the device to different wavelengths and power density and its response time could be measured by introducing the fiber laser into the 4200 probe system. Photoresponsivity, external quantum efficiency and detection rate can be calculated based on these original data. The power of fiber laser can be modulated continuously from 0 to $28 \mathrm{~mW}$, and the spot diameter is $1 \mathrm{~mm}$.

\section{Results and Discussion}

Characterization of few-layer $W_{2}$ Nanoflakes and FETs: Figure 1(a) shows the AFM scanning image of our device, and the thickness of $\mathrm{WS}_{2}$ is approximately 3nm, confirmed by AFM (figure 1(b). As known to all, the interlayer spacing of $\mathrm{WS}_{2}$ is $0.7 \mathrm{~nm}$, thus we judged that the sample used in the experiment was approximately 4 layer. Figure 1(c) demonstrates the optical image of the device. As shown in the figure, the channel length is 3.979um and channel width is $1.957 \mathrm{um}$. Figure $1(\mathrm{~d})$ and figure 1(c) illustrates the schematic diagram of back-gated few-layer $\mathrm{WS}_{2} \mathrm{FET}$, wherein the electrodes are composed of $20 \mathrm{~nm} \mathrm{Ti}$ and $70 \mathrm{~nm} \mathrm{Au}$. By applying the $\mathrm{Si} / \mathrm{SiO}_{2}$ substrate as back-gate, the Ids of the device could be regulated through changing the magnitude of base voltage.
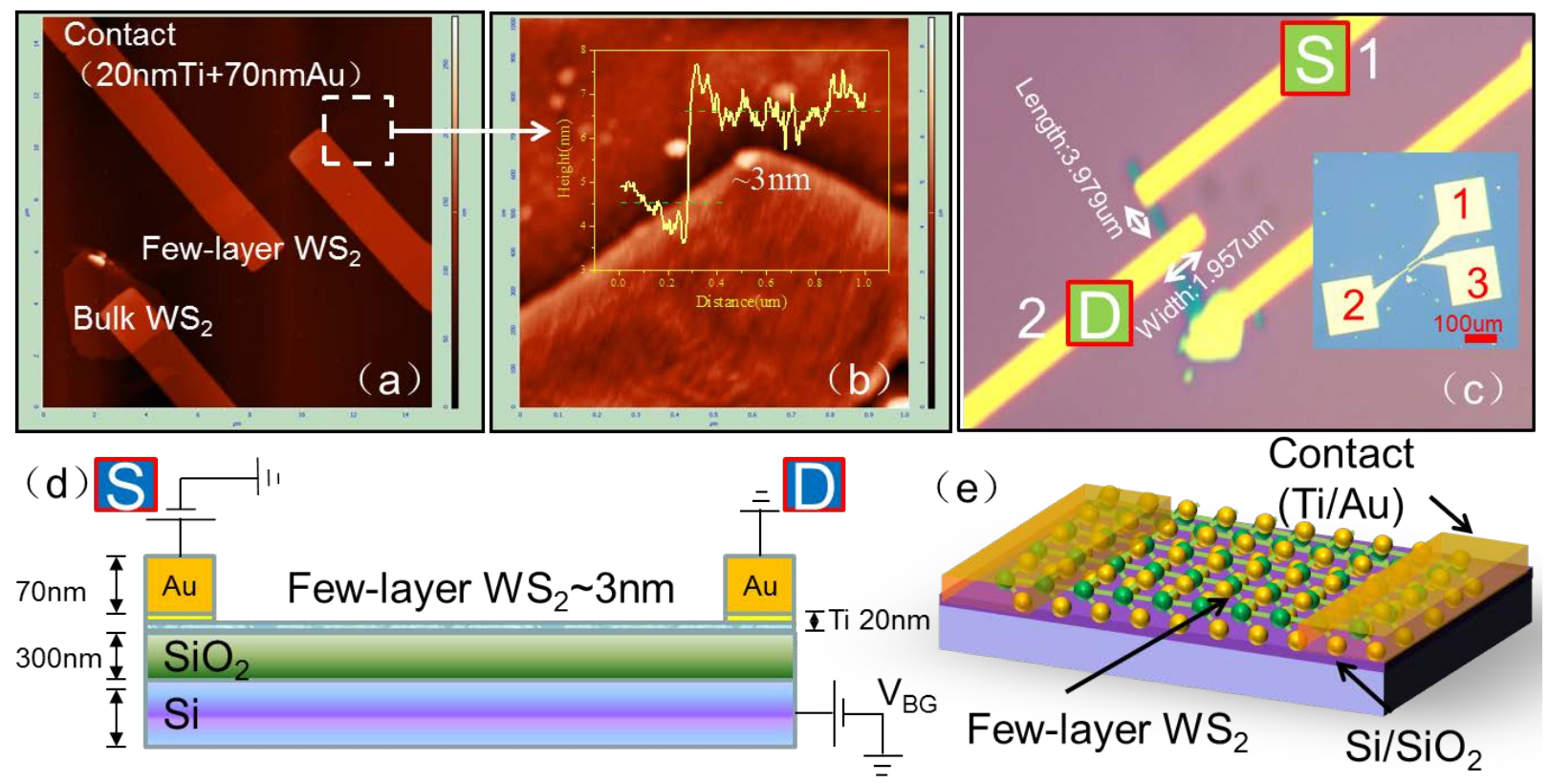

Figure 1. Characterization of few-layer $\mathrm{WS}_{2}$ Nanoflakes and FETs.(a)AFM scanning image of few-layer $\mathrm{WS}_{2}$ nanoflakes FET(b) Terrace height of $\mathrm{WS}_{2}$ nanoflakes(c)Optic image of fabricated few-layer $\mathrm{WS}_{2}$ FET. The inset shows the image observed by microscope in a lower magnification. (d) Side elevation of fabricated back-gated few-layer $\mathrm{WS}_{2}$ FET, highly p-doped silicon serves as back gate.(e) Three-dimensional schematic of the typical device structure. 
Fundamental Electrical Properties: Figure 2 illsultrates the I-V characterastics of few-layer WS 2 nanoflakes FET in high bias voltage(a) and low bias voltage(b). As shown in figure 2(a), the Ids increases when applied a higher backgate voltage. This is because the Fermi level of $\mathrm{WS}_{2}$ is improved by electron dopping from $\mathrm{Si} / \mathrm{SiO}_{2}$ bakegate structure. Apperantly, the current in negative bias is different from the current in positive bias, which indicates an asymmertrical semi-contact. Linear region exists when applied low bias voltage. Figure 2(b) shows the output characterastics when bias voltage sweeps from $-0.1 \mathrm{~V}$ to $+0.1 \mathrm{~V}$. It is clear that the current is linearly dependent on bias voltage, which means the semi-contact barrier does not have a significant influence on device in low bias. Noticeably, the dark resistance of few-layer $\mathrm{WS}_{2}$ nanoflakes is extremely high, amazingly reaching hundreds $G \Omega$ (when Vbg=0V). The high resistance mainly results from the low carrier density of this typical material[16, 17].
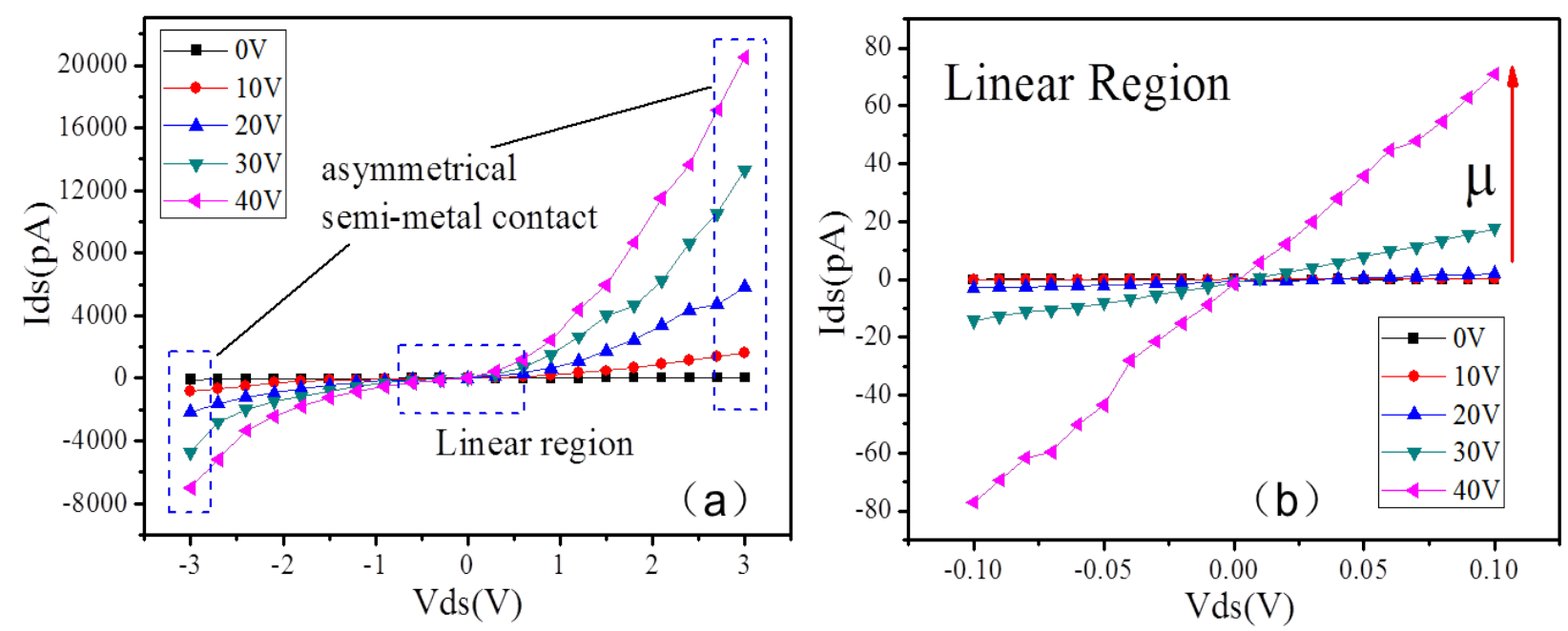

Figure 2. I-V characterastics of few-layer $\mathrm{WS}_{2}$ nanoflakes FET. (a)The output characteristics of the device when applyed different backgate voltage ( $0 \mathrm{~V}$ to $40 \mathrm{~V})$, bias voltage sweeps from -3V to $+3 \mathrm{~V}$.(b)Linear reagion of the device when applied low bias voltage $(-0.1 \mathrm{~V}$ to $+0.1 \mathrm{~V})$.

The transfer characteristic curves are shown in figure 3(a) and figure 3(b). The electron mobility $\mu$ can be obtained from the equation[10]:

$\mu=\frac{\partial I_{D S}}{\partial V_{G}}\left(\frac{L}{W C_{i} V_{D S}}\right)$

where $L$ is the channel length $(3.979 u m), W$ is the channel width $(1.957 \mathrm{um})$, and $C_{i}$ is the gate capacitance which can be given by the equation $C_{i}=\varepsilon_{0} \varepsilon_{r} / d . \varepsilon_{0}\left(8.8510^{-12} \mathrm{~F} / \mathrm{m}\right)$ is vacuum dielectric constant, and $\varepsilon_{r}$ (3.9) and $d(300 \mathrm{~nm})$ are dielectric constant and thickness of $\mathrm{SiO}_{2}$ respectively. 

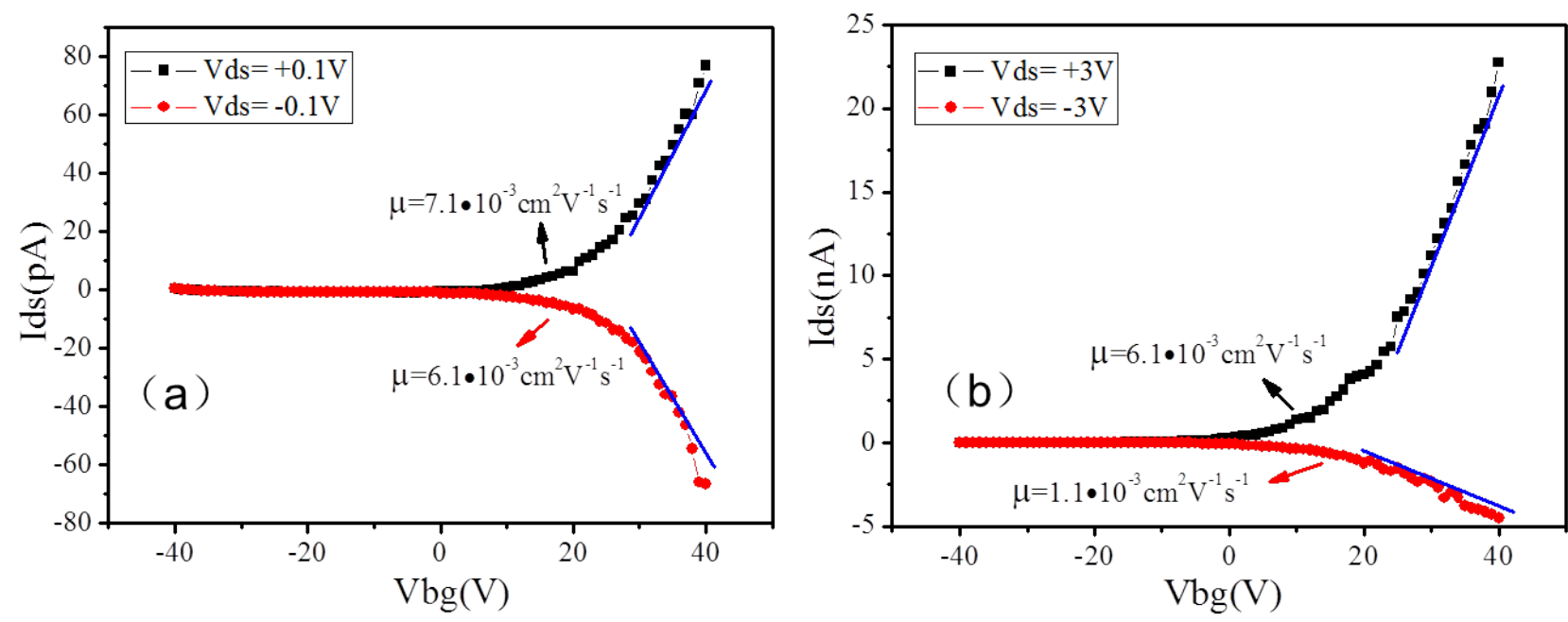

Figure 3. Transfer characterastics of few-layer $\mathrm{WS}_{2}$ nanoflakes FET. The gate voltage varies from $-40 \mathrm{~V}$ to $+40 \mathrm{~V}$ when bias voltage is a fixed alue .(a) $\mathrm{Vds}=-0.1 \mathrm{~V}$ and $+0.1 \mathrm{~V}$. (b) Vds=-3V and $+3 \mathrm{~V}$.

According to calculation, the electron mobility is $7.1 \cdot 10^{-3} \mathrm{~cm}^{2} \mathrm{~V}^{-1} \mathrm{~s}^{-1}(\mathrm{Vds}=+0.1 \mathrm{~V})$ and $6.1 \cdot 10^{-3} \mathrm{~cm}^{2} \mathrm{~V}^{-1} \mathrm{~s}^{-1}(\mathrm{Vds}=-0.1 \mathrm{~V})$. However, when applied high bias voltage, the electron mobility has a signifancant difference in positive bias $\left(6.1 \cdot 10^{-3} \mathrm{~cm}^{2} \mathrm{~V}^{-1} \mathrm{~s}^{-1}\right)$ and negative bias $\left(1.1 \cdot 10^{-3} \mathrm{~cm}^{2} \mathrm{~V}^{-1} \mathrm{~s}^{-1}\right)$. This is mainly because the asymmertrical semi-contact, which is in accordance with the previous measurement data(figure 2(a)). Besides, the On/Off ratio may reaches amazingly $10^{4}$ in few-layer $\mathrm{WS}_{2}$ nannoflakes FET calculated by the experimental data. Accordingly, although the mobility of few-layer $\mathrm{WS}_{2}$ is lower than graphene, however, it possesses extrodinary On/Off ratio, thus $\mathrm{WS}_{2}$ is more promising to be applied in the logic operation device compared to the graphene.

As previous research reported[9], $\mathrm{WS}_{2}$ generally exhibit an n-type conductivity type.Similayerly, the $\mathrm{WS}_{2}$ device we prepared also demonstrated an n-type property. This phenomenon is owing to Se vacancies in the process of synthesis.

Photo-electrical properties: Bulk WS 2 is an indirect-bandgap $(1.4 \mathrm{eV})$ semiconductor, but can turn into a direct-bandgap $(2.1 \mathrm{eV})$ material when exfoliated into the monolayer state[9]. Unlike bulk or multi-layer $\mathrm{WS}_{2}$, few-layer still demonstrates an intense optical excitation. According to the theoretical analysis[9], the few-layer $\mathrm{WS}_{2}$ nanoflakes can respond to the visible light. Thus, $638 \mathrm{~nm}$ laser light was exerted to investigate the photo-electrical properties of $\mathrm{WS}_{2}$. For convenience, the laser power showed in figure measured by photo-power meter (THORLABS PM100A) is total power of incident laser rather than the light on the device.
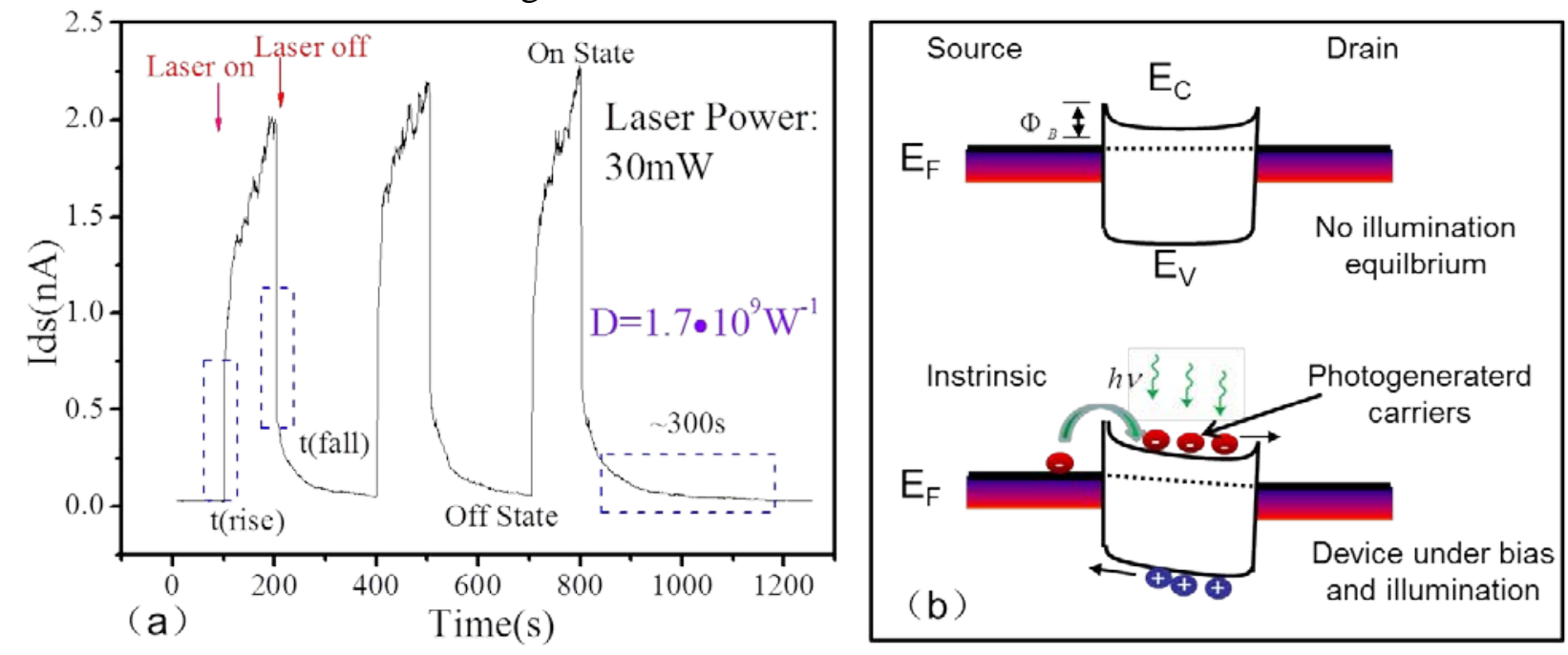

Figure 4. Photo-response of few-layer $\mathrm{WS}_{2}$ optoelectronical device.(a) Time-resolved photoresponse of the device, during the light switching on/off at source drain voltage of $+3 \mathrm{~V}(\mathrm{Vbg}=0 \mathrm{~V}$. (b) Band 
diagram of the few-layer $\mathrm{WS}_{2}$ photo-detector taking into consideration Schottky barriers at the contacts. $E_{F}$ is the Fermi level energy, $E_{C}$ the minimum conduction band energy, $E_{V}$ the maximum valence band energy and $\Phi_{B}$ the Schottky barrier height.

The device exhibits fast dynamic response for both rise and decay process (figure 5(a)), the response and recovery time (blue dotted line frame) is shorter than the detection limit of our measurement setup (20ms). Disapointedly, owing to the existing of defect state, the electron-hole pairs can not be seperated and recombined at its instrinsic properaty. As seen in the figure, the photocurrent continuiously grows up when exerted laser irriadition. Besides, when laser is turned off, the device needs approximately 300s to recombine all the electron-hole pairs in defect state. However, it is of no significant influence on detecting light. Here, we introduce the detection rate, defined as:

$$
D=\frac{I_{\text {on }} / I_{\text {off }}}{P_{\text {in }}}
$$

This parameter can well characterize the $\mathrm{S} / \mathrm{N}$ (signal to noise) ratio of photoresponse of the device. Owing to the dark resistance of device is particularly large, the device based on $\mathrm{WS}_{2}$ showed excellent performance in detection. By calculation, the detection rate of the device reaches up to highly $1.7 \cdot 10^{9}$ $W^{1}$, showing its good switching performance, which could be effectively applied in measuring the photo-electric signal.

The behavior of photo-generated charge-carriers of photodetector can be explained by a simple energy band diagram (figure 4(b)). With no illumination and without applying gate or drain bias, the device is in its equilibrium state, characterized by Schottky barriers at the contacts[18]. Illuminating the device with laser light, resulting in light absorption and excitation of electron-hole pairs, which can be extracted by applying a drain-source bias[13, 18, 19].

Figure 5(a) demonstrates the Ids-Vds characteristics under different laser power, where the drain current increased when the laser power was enhanced at a fixed drain-source voltage $(-3 \mathrm{~V}$ and $+3 \mathrm{~V})$ due to the growth in the photogenerated current with the increased laser power.
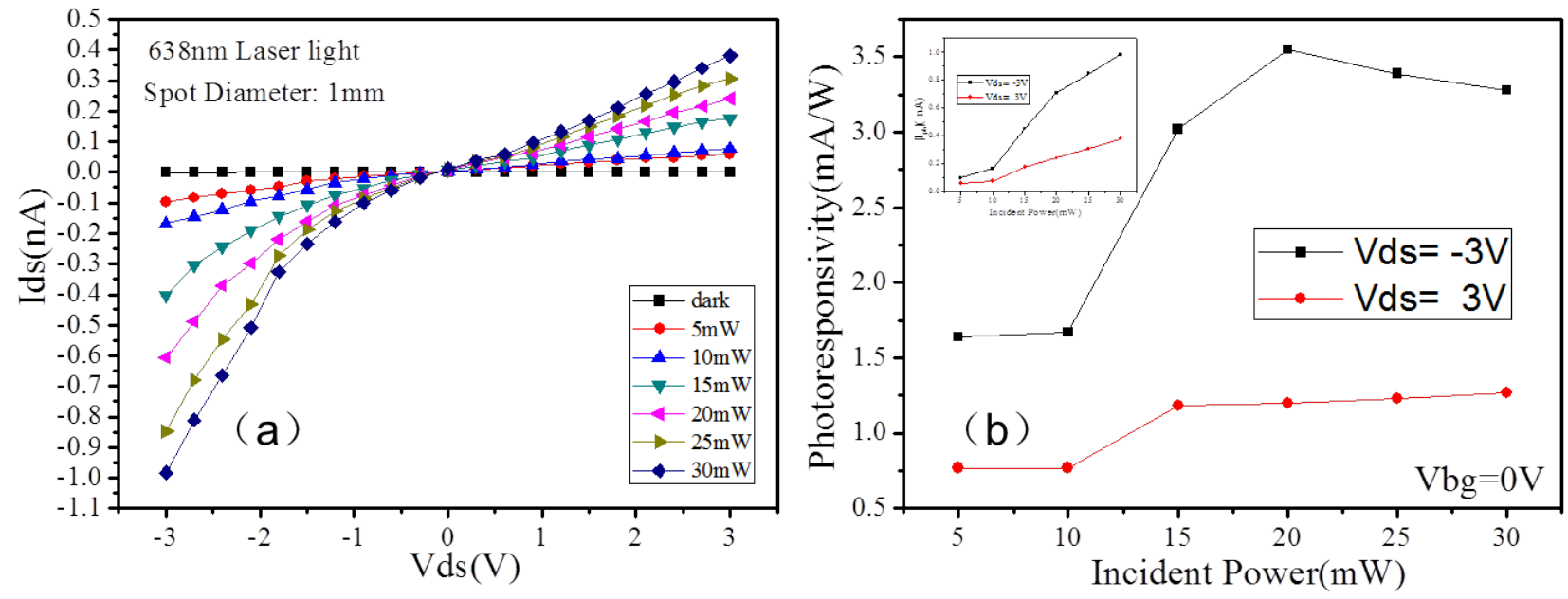

Figure 5. Photo-response of few-layer $\mathrm{WS}_{2}$ optoelectronical device. (a)Drain-source (Ids-Vds characteristic of the device under different illumination intensities.(b) The dependence of photo-current upon the laser power when $\mathrm{V}_{\mathrm{DS}}=-3 \mathrm{~V}$ and $+3 \mathrm{~V}$. The inset is the photo-responsivity obtained by calculating.

Photoresponsivity is defined by the equation $R_{\lambda}=I_{p h} / P_{\text {in }}$, where $I_{p h}=I_{\text {on }}-I_{\text {off }}$ ( $I_{\text {on }}$ and $I_{\text {off }}$ represent photo current and dark current respectively), and $P_{\text {in }}=P_{\text {laser }} \square\left(S_{\text {area }} / S_{\text {spot }}\right)$. Therefore, we applied the equation to calculate the photoresponsivity.

$$
R_{\lambda}=\frac{I_{\text {on }}-I_{\text {off }}}{P_{\text {laser }}\left(S_{\text {area }} / S_{\text {spot }}\right)}
$$


According to the data in optic image (figure 1(c)), the photosensitive area of the device $S_{\text {area }}=L \cdot d$, where $L=3.979 u m, d=1.957 \mathrm{um}$, meaning that the actual working area to be $7.787 \mathrm{um}^{2}$. Furthermore, $S_{\text {spot }}=0.785 \mathrm{~mm}^{2}$ owing to the spot diameter is $1 \mathrm{~mm}$.

Figure 4(b) shows the photoreponsivity of various laser power when Vds $=-3 \mathrm{~V}$ and $\mathrm{Vds}=+3 \mathrm{~V}$. The inset shows the photogenerated current was proportional to the drain-source voltage at a fixed laser power. The photoreponsivity of positive area is continuously larger than which in negative area due to asymmertrical semi-contact. In general, the lower limit of the photo responsivity is determined by the dark resistance and the responsivity will be maintained at a constant value as photocurrent increase until the light saturation[20]. The photorponsivity of the fabricated device in experiment can surprisingly attain $3.5 \mathrm{~mA} / \mathrm{W}(\mathrm{Vds}=+3 \mathrm{~V}$, laser power $=20 \mathrm{~mW})$, which means a potential in the practical application.

As for the practical application of the photo-detector, a highly significant parameter is the external quantum efficiency (EQE) $\eta(\lambda)$.When photo-response lies in linear region, external quantum efficiency can be obtained by the equation:

$$
\eta(\lambda)=\frac{R_{\lambda} h c}{q \lambda}
$$

where $R_{\lambda}$ is photoresponsivity, $h$ on behalf of Planck constant $\left(6.62 \cdot 10^{-34} \mathrm{~J} \cdot \mathrm{s}\right), c$ the velocity of light $\left(3 \cdot 10^{8} \mathrm{~m} / \mathrm{s}\right), q$ the elementary charge of electricity $\left(1.6 \cdot 10^{-19} \mathrm{C}\right)$ and $\lambda$ the wavelength of the incident light. According to the experimental data, the $\eta(\lambda)$ of fabricated device could reach as high as $0.68 \%$ $(\mathrm{Vds}=+3 \mathrm{~V}, \mathrm{Vbg}=0 \mathrm{~V})$ when responding to red light.

\section{Summary}

To summarize, we have fabricated phototransistors based on few-layer $\mathrm{WS}_{2}$. Due to the unique structural properties of two-dimensional material, devices based on it exhibit a high photo-electrical properties. The device demonstrate a high On/Off ratio $\left(\sim 10^{4}\right)$ and relatively high carrier mobility $\left(7.110^{-3} \mathrm{~cm}^{2} V^{-1} \mathrm{~s}^{-1}\right)$. In our experiment, $638 \mathrm{~nm}$ laser light was systematically investigated. Performance parameters, such as photoresponsivity $\left(R_{\lambda}=3.5 \mathrm{~mA} / \mathrm{W}\right)$, response time $(t<20 \mathrm{~ms})$, external quantum efficiency $(\eta=0.68 \%)$, detection rate $\left(D=1.7 \times 10^{9} \mathrm{~W}^{-1}\right)$ and the photocurrent as a function of incident power were studied. Combining our results with large-area material preparation methods such as liquid scale exfoliation or CVD growth, together with the simplicity of the device presented here, could also result in the fabrication of inexpensive, high-sensitivity and flexible few-layer $\mathrm{WS}_{2}$ optoelectronic devices. Possible fields of applications could include consumer imaging sensors suitable for low-light photography, for example in cell-phone cameras, which currently suffer from poor low-light performance, or in sensors for fluprescence imaging.

\section{Acknowledgement}

This work was supported by the National Natural Science Foundation of China (No. 11574395), the Research Programme of National University of Defense Technology (No. JC15-02-01), the open foundation of State Key Laboratory of high Performance Computing (No. 201301-02), and the open foundation based on the innovation platform of Hunan key laboratories (No. 13K022).

\section{References}

[1] Novoselov KS, Geim AK, Morozov S, Jiang D, Zhang Y, Dubonos Sa, et al. Electric field effect in atomically thin carbon films. science. 2004;306(5696):666-9.

[2] Abajo FJGD, Koppens FHL, Chang DE, Thongrattanasiri S, editors. Graphene Plasmonics. Fourth International Workshop on Theoretica; 2011. 
[3] Bertolazzi S, Krasnozhon D, Kis A. Nonvolatile memory cells based on MoS2/graphene heterostructures. ACS nano. 2013;7(4):3246-52.

[4] Novoselov KS, Jiang Z, ., Zhang Y, ., Morozov SV, Stormer HL, Zeitler U, ., et al. Room-temperature quantum Hall effect in graphene. Science. 2007;315(5817):1379-.

[5] Xia F, Mueller T, Lin Y-m, Valdes-Garcia A, Avouris P. Ultrafast graphene photodetector. Nature nanotechnology. 2009;4(12):839-43.

[6] Geim AK, Novoselov KS. The rise of graphene. Nature materials. 2007;6(3):183-91.

[7] Geim AK. Graphene: status and prospects. Science. 2009;324(5934):1530-4.

[8] Gutiérrez HR, Perea-López N, Elías AL, Berkdemir A, Wang B, Lv R, et al. Extraordinary room-temperature photoluminescence in triangular WS2 monolayers. Nano letters. 2012;13(8):3447-54.

[9] Cong C, Shang J, Wu X, Cao B, Peimyoo N, Qiu C, et al. Synthesis and Optical Properties of Large - Area Single - Crystalline 2D Semiconductor WS2 Monolayer from Chemical Vapor Deposition. Advanced Optical Materials. 2014;2(2):131-6.

[10] Huo N, Yang S, Wei Z, Li S-S, Xia J-B, Li J. Photoresponsive and gas sensing field-effect transistors based on multilayer WS2 nanoflakes. Scientific reports. 2014;4.

[11] Zhang Y, Zhang Y, Ji Q, Ju J, Yuan H, Shi J, et al. Controlled growth of high-quality monolayer WS2 layers on sapphire and imaging its grain boundary. ACS nano. 2013;7(10):8963-71.

[12] Geim A, Novoselov K. The rise of graphene. naturematerials, 6: 183-191. March; 2007.

[13] Radisavljevic B, Radenovic A, Brivio J, Giacometti V, Kis A. Single-layer MoS2 transistors. Nature nanotechnology. 2011;6(3):147-50.

[14] Yu JH, Lee HR, Hong SS, Kong D, Lee H-W, Wang H, et al. Vertical Heterostructure of Two-Dimensional MoS2 and WSe2 with Vertically Aligned Layers. Nano letters. 2015;15(2):1031-5.

[15] Tonndorf P, Schmidt R, Böttger P, Zhang X, Börner J, Liebig A, et al., editors. Photoluminescence Emission and Raman Response of MoS2, MoSe2, and WSe2 Nanolayers. CLEO: QELS_Fundamental Science; 2013: Optical Society of America.

[16] Allain A, Kang J, Banerjee K, Kis A. Electrical contacts to two-dimensional semiconductors. Nature Materials. 2015;14(12):1195-205.

[17] Liu W, Kang J, Sarkar D, Khatami Y, Jena D, Banerjee K. Role of metal contacts in designing high-performance monolayer n-type WSe2 field effect transistors. Nano letters. 2013;13(5):1983-90.

[18] Lopez-Sanchez O, Lembke D, Kayci M, Radenovic A, Kis A. Ultrasensitive photodetectors based on monolayer MoS2. Nature nanotechnology. 2013;8(7):497-501.

[19] Tsai D-S, Liu K-K, Lien D-H, Tsai M-L, Kang C-F, Lin C-A, et al. Few-layer MoS2 with high broadband photogain and fast optical switching for use in harsh environments. Acs Nano. 2013;7(5):3905-11.

[20] Ross JS, Klement P, Jones AM, Ghimire NJ, Yan J, Mandrus D, et al. Electrically tunable excitonic light-emitting diodes based on monolayer WSe2 pn junctions. Nature nanotechnology. 2014;9(4):268-72. 